\title{
Intermittent post-exercise sauna bathing improves markers of exercise capacity in hot and temperate conditions in trained middle-distance runners
}

\author{
Nathalie V. Kirby ${ }^{1}$ (D) Samuel J. E. Lucas ${ }^{1}$ - Oliver J. Armstrong ${ }^{2} \cdot$ Samuel R. Weaver $^{1} \cdot$ Rebekah A. I. Lucas $^{1}$
}

Received: 6 April 2020 / Accepted: 24 October 2020 / Published online: 19 November 2020

(c) The Author(s) 2020

\begin{abstract}
Purpose This study investigated whether intermittent post-exercise sauna bathing across three-weeks endurance training improves exercise heat tolerance and exercise performance markers in temperate conditions, compared to endurance training alone. The subsidiary aim was to determine whether exercise-heat tolerance would further improve following 7-Weeks post-exercise sauna bathing.

Methods Twenty middle-distance runners ( 13 female; mean $\pm \mathrm{SD}$, age $20 \pm 2$ years, $V \mathrm{O}_{2 \max } 56.1 \pm 8.7 \mathrm{ml} \mathrm{kg}^{-1} \mathrm{~min}^{-1}$ ) performed a running heat tolerance test $\left(30-\mathrm{min}, 9 \mathrm{~km} \mathrm{~h}^{-1} / 2 \%\right.$ gradient, $40{ }^{\circ} \mathrm{C} / 40 \% \mathrm{RH}$; HTT) and temperate $\left(18{ }^{\circ} \mathrm{C}\right)$ exercise tests (maximal aerobic capacity $\left[V \mathrm{O}_{2 \mathrm{max}}\right]$, speed at $4 \mathrm{mmol} \mathrm{L}{ }^{-1}$ blood lactate concentration ([ $\left.\mathrm{La}^{-}\right]$) before (Pre) and following three-weeks (3-Weeks) normal training (CON; $n=8)$ or normal training with $28 \pm 2$ min post-exercise sauna bathing $\left(101-108{ }^{\circ} \mathrm{C}, 5-10 \% \mathrm{RH}\right) 3 \pm 1$ times per week (SAUNA; $n=12$ ). Changes from Pre to 3-Weeks were compared betweengroups using an analysis of co-variance. Six SAUNA participants continued the intervention for 7 weeks, completing an additional HTT (7-Weeks; data compared using a one-way repeated-measures analysis of variance).

Results During the HTT, SAUNA reduced peak rectal temperature $\left(T_{\text {rec }} ;-0.2{ }^{\circ} \mathrm{C}\right)$, skin temperature $\left(-0.8{ }^{\circ} \mathrm{C}\right)$, and heart rate $\left(-11\right.$ beats $\left.\mathrm{min}^{-1}\right)$ more than $\mathrm{CON}$ at 3 -Weeks compared to Pre (all $\left.p<0.05\right)$. SAUNA also improved $V \mathrm{O}_{2 \max }(+0.27$ $\left.\mathrm{L}^{-1} \min ^{-1} ; p=0.02\right)$ and speed at $4 \mathrm{mmol} \mathrm{L}^{-1}\left[\mathrm{La}^{-}\right]\left(+0.6 \mathrm{~km} \mathrm{~h}^{-1} ; p=0.01\right)$ more than CON at 3-Weeks compared to Pre. Only peak $T_{\text {rec }}\left(-0.1^{\circ} \mathrm{C} ; p=0.03\right.$ decreased further from 3-Weeks to 7-Weeks in SAUNA (other physiological variables $p>0.05$ ).

Conclusions Three-weeks post-exercise sauna bathing is an effective and pragmatic method of heat acclimation, and an effective ergogenic aid. Extending the intervention to seven weeks only marginally improved $T_{\text {rec }}$.
\end{abstract}

Keywords Post-exercise sauna $\cdot$ Thermoregulation $\cdot$ Exercise performance $\cdot$ Heat acclimation $\cdot$ Ergogenic aid

\section{Abbreviations}

3-Weeks Tests following three-weeks intervention

7-Weeks Tests following seven-Weeks intervention

ANOVA Analysis of variance

Communicated by Narihiko Kondo.

Electronic supplementary material The online version of this article (https://doi.org/10.1007/s00421-020-04541-z) contains supplementary material, which is available to authorized users.

Nathalie V. Kirby

NXK650@bham.ac.uk

University of Birmingham, Birmingham B15 2TT, UK

2 Performance Centre, University of Birmingham Sport, Birmingham, UK

$\begin{array}{ll}\text { ANCOVA } & \text { Analysis of co-variance } \\ \text { CON } & \text { Control group } \\ \text { EPO } & \text { Erythropoietin } \\ \text { HR } & \text { Heart rate } \\ \text { HTT } & \text { Running heat tolerance test } \\ \text { Pre } & \text { Pre-intervention tests } \\ \text { RER } & \text { Respiratory exchange ratio } \\ \text { RPE } & \text { Rating of perceived exertion } \\ \text { SAUNA } & \text { Sauna intervention group } \\ T_{\text {rec }} & \text { Rectal temperature } \\ T_{\text {sk }} & \text { Mean weighted skin temperature } \\ \text { TTE } & \text { Time-to-exhaustion } \\ \text { VEGF } & \text { Vascular endothelial growth factor } \\ V \mathrm{O}_{2 \mathrm{max}} & \text { Maximal aerobic capacity }\end{array}$




\section{Introduction}

Heat acclimation improves exercise performance in the heat (Sawka et al. 2011). The most common model studied in the literature is "medium-term" active heat acclimation (Tyler et al. 2016), where individuals exercise in a climatic/environmental chamber for 60-120 min for 7-14 consecutive days (Garrett et al. 2009). This model of heat acclimation carries considerable barriers related to financial and temporal costs, as well as accessibility to climatic/environmental chambers. Consequently, heat acclimation is not widely used by athletes (recently recorded prevalence of $\sim 15 \%$; Périard et al. 2015) despite its effectiveness in improving exercise performance in hot environments. With major athletic events in extreme heat fast approaching, such as the Tokyo 2020 Olympic Games (postponed; Gerrett et al. 2019) and the 2022 FIFA World Cup in Doha, it is imperative that athletes are prepared to compete in such challenging environments. Post-exercise sauna bathing presents a practical and accessible heat acclimation alternative to active heat acclimation that could be implemented without disruption to an athlete's training programme. Repeated bouts of sauna bathing have been observed to elicit some heat acclimation adaptations [e.g., reduced resting core temperature with sauna independent of exercise (Leppäluoto et al. 1986) and plasma volume expansion using post-exercise sauna bathing (Scoon et al. 2007; Stanley et al. 2015)]. However, its efficacy to elicit hallmark heat acclimation adaptations during exercise heat stress (e.g., reduced exercising heart rate and body temperatures, etc.; Sawka et al. 2011) has not previously been investigated (Casadio et al. 2017). Despite the relatively sparse scientific evidence, post-exercise sauna bathing is recommended to athletes preparing for competition in the heat (Racinais et al. 2019). It more specifically recommends that ahtletes use post-exercise sauna bathing to prepare for competition in the heat than the reference currently used. Therefore, the first aim of this study was to assess exercise heat tolerance following repeated bouts of intermittent sauna bathing using a fixed-workload exercise heat stress test.

The myriad of physiological adaptations attained through heat acclimation may improve exercise performance in cool or temperate conditions (Minson and Cotter 2016). Similar improvements may result from post-exercise sauna bathing, as a case study of a young female tennis player showed improvements in performance outcomes of timeto-exhaustion (TTE), maximal aerobic capacity $\left(V \mathrm{O}_{2 \max }\right)$, and lactate threshold after 12 sauna sessions following exercise spread across 3 weeks (Novak et al. 2018). Similarly, Scoon and colleagues (2007) observed that running TTE increased in six male athletes after approximately 12 intermittent post-exercise sauna sessions. However, the effects of intermittent post-exercise sauna bathing on more objective markers of temperate exercise performance (i.e., $V \mathrm{O}_{2 \max }$ and blood lactate profile) have not been assessed in a larger cohort. Therefore, the second aim of this study was to assess whether integrating intermittent post-exercise sauna bathing $\left(\sim 3\right.$ sessions $\left.\cdot w^{-} \mathrm{e}^{-1}\right)$ into an endurance training programme further improves temperate exercise performance markers $\left(V \mathrm{O}_{2 \max }\right.$ and running speed at $4 \mathrm{mmol} \mathrm{L}{ }^{-1}$ blood lactate concentration $\left.\left[\mathrm{La}^{-}\right]\right)$, as compared to an endurance training programme alone.

Finally, long-term heat acclimation interventions (i.e., $>14$ days) are expected to maximise exercise capacity in the heat (Tyler et al. 2016). These longer protocols may also allow time for the increased production of erythropoietin (EPO) and vascular endothelial growth factor (VEGF), as observed following hypoxia exposure (Girard et al. 2013), both primary factors driving favourable adaptations to oxygen carrying capacity and delivery. Notably, 10 consecutive days of active heat acclimation elicits increases in hypoxia inducible factor 1-alpha (HIF-1a; the master regulator of downstream oxygen-modulating genes such as EPO and VEGF) in males (Lee et al. 2016). Therefore, the subsidiary aim of this study was to assess exercise heat tolerance and changes in EPO and VEGF in response to long-term (i.e., more than 14 heat exposures) intermittent post-exercise sauna bathing.

The purpose of this study was to determine the efficacy of adding intermittent post-exercise sauna bathing to normal endurance training to induce heat acclimation adaptations in trained athletes, and to determine the impact of these adaptations on maximal and submaximal exercise performance markers in temperate conditions. We hypothesised that intermittent post-exercise sauna bathing would: (1) elicit hallmark heat acclimation adaptations and improve thermoregulation during exercise heat stress more than endurance training alone, and (2) improve physiological markers of exercise performance in temperate conditions to a greater extent than endurance training alone.

\section{Methods}

\section{Ethical approval}

This study was approved by the University of Birmingham Ethics Committee (ERN_18-0958), and conformed to the standards set by the Declaration of Helsinki. All participants were informed of the experimental procedures and possible risks involved in the study before providing written consent.

\section{General overview and design}

Each participant completed a general health questionnaire and female participants completed a menstrual cycle 
questionnaire. Throughout the protocol, participants were asked to keep a training diary (including session type, running distance, frequency and session perceived exertion) and female participants were asked to record their menstrual cycle. An overview of the protocol is depicted in Fig. 1. Participants took daily iron tablets $(65 \mathrm{mg}$ ferrous sulfate; Nature Made, West Hills, CA, United States) from 2 weeks prior to the first experimental trial until the completion of the protocol.

A battery of experimental trials was completed within a 1-week timeframe at baseline (Pre), following 3 weeks (3-Weeks) and following 7 weeks (7-Weeks) of either normal endurance training $(\mathrm{CON})$ or normal endurance training with the post-exercise sauna bathing intervention (SAUNA). Experimental trials included a running heat tolerance test (HTT), a temperate exercise test (consisting of a lactate profile test to determine running speed at $4 \mathrm{mmol} \mathrm{L}^{-1}\left[\mathrm{La}^{-}\right]$and a $V \mathrm{O}_{2 \max }$ test) and a resting venous blood sample. The temperate exercise test was conducted at Pre and 3-Weeks only.

Experimental trials following three-and seven-weeks intervention were arranged $\sim 28$ days apart to test female participants during the same menstrual cycle phase, as per a typical menstrual cycle. Participants self-allocated into groups prior to Pre testing. This experiment was conducted in the United Kingdom between the months of October and March to minimise any natural heat acclimatisation.

\section{Participants}

Fifty-one trained middle-distance and cross-country runners were recruited from the university's athletics club to participate in this study. However, due to various reasons (injury, scheduling, etc.) only 20 athletes (SAUNA, $n=12$ [F, $n=9$; $\mathrm{M}, n=3]$; CON, $n=8$ [F, $n=4$; M, $n=4]$ ) completed experimental trials at 3-Weeks. Two female participants completed both the SAUNA and CON interventions (in opposite order, with $\sim 5$ weeks washout), one 'cross-over' participant only completing the Lactate Profile test at both Pre and 3-Weeks whilst undergoing the $\mathrm{CON}$ intervention. Eight athletes completed experimental trials at 7-weeks, though only the six athletes in the SAUNA group $(\mathrm{F}, n=3 ; \mathrm{M}, n=3)$ will be reported in the HTT at the final time-point (7-Weeks). The two athletes in the CON group who completed experimental trials at 7-Weeks were not included in the 7-Weeks analysis. An overview of the progression of athletes through the study is detailed in Fig. 2. Participants in the SAUNA and CON group at 3-Weeks had similar baseline characteristics (age $19 \pm 1$ and $20 \pm 2$ years, height $169 \pm 7$ and $172 \pm 6 \mathrm{~cm}, \dot{V}$ $\mathrm{O}_{2 \max } 59.3$ and $58.4 \pm 7.2 \mathrm{ml} \mathrm{kg}^{-1} \mathrm{~min}^{-1}$, respectively; all $p>0.05)$. SAUNA participants who completed experimental trials at 7-Weeks were $19 \pm 1$ years of age, $171 \pm 6 \mathrm{~cm}$ tall and had a baseline $\dot{V} \mathrm{O}_{2 \max }$ of $60.2 \pm 10.1 \mathrm{ml} \mathrm{kg}^{-1} \mathrm{~min}^{-1}$. Female participants included in analyses at 3-Weeks were eumenorrheic $(n=7)$, oligomenorrheic $(n=1)$, amenorrheic $(n=1)$, or using various forms of hormonal contraceptives (monophasic oral contraceptive pill, $n=1$; progestin-only oral contraceptive pill, $n=1$; contraceptive coil, $n=1$ ). Of these, female participants included in analysis at 7-Weeks were either eumenorrheic $(n=2)$ or amenorrheic $(n=1)$. Female participants did not report any negative menstrual or Pre-menstrual symptoms that may have affected performance (Giacomoni et al. 2000).

\section{Post-exercise sauna bathing}

Participants entered the sauna $\left(101-108{ }^{\circ} \mathrm{C}\right.$ at $\sim 1.8 \mathrm{~m}$, 5-10\% RH at 1 m; iButton Hygrochron Logger, Maxim Integrated, California, USA) within $\sim 5$ min of cessation of outdoor exercise. Sauna bathing typically followed lowintensity, continuous exercise training sessions (i.e., 'easy runs' or 'long runs'). Participants were asked to remain in the sauna for 30-min, as per previous investigations (Scoon et al. 2007; Stanley et al. 2015). In the sauna, participants sat upright and were allowed to drink ad libitum. Peak heart rate (HR) was recorded during the final minute of exposure.

\section{Experimental trials}

Participants performed all experimental trials at the same time of day $( \pm 2 \mathrm{~h})$. Participants were asked to recall the

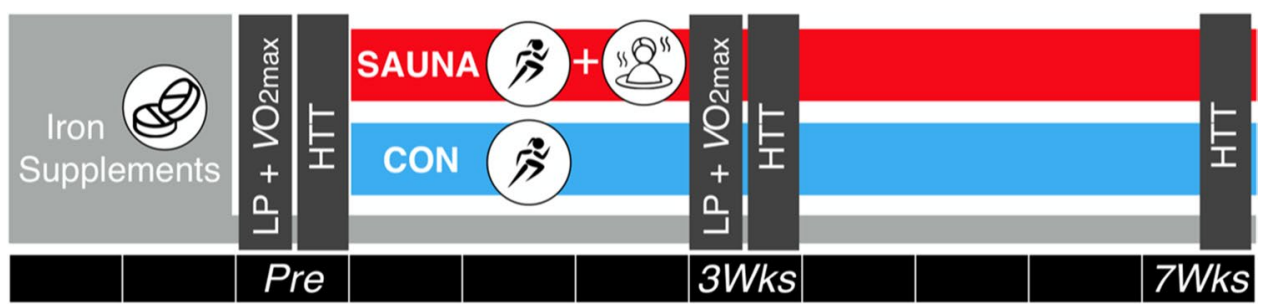

Fig. 1 Schematic of the experimental design. Post-exercise sauna bathing intervention group (SAUNA) and control group (CON) completed temperate exercise tests $\left(18{ }^{\circ} \mathrm{C}\right)$ consisting of lactate profile (LP) and maximal aerobic capacity $\left(V \mathrm{O}_{2 \max }\right)$ tests, and a running heat tolerance test (HTT; 30-min, $9 \mathrm{~km} \mathrm{~h}^{-1} / 2 \%$ gradient, $40{ }^{\circ} \mathrm{C} / 40 \% \mathrm{RH}$ ), at baseline (Pre), and following 3 -Weeks (3Wks) intervention or control. Participants completed an additional HTT following 7-Weeks intervention (7Wks). Lower black bars indicate weeks 


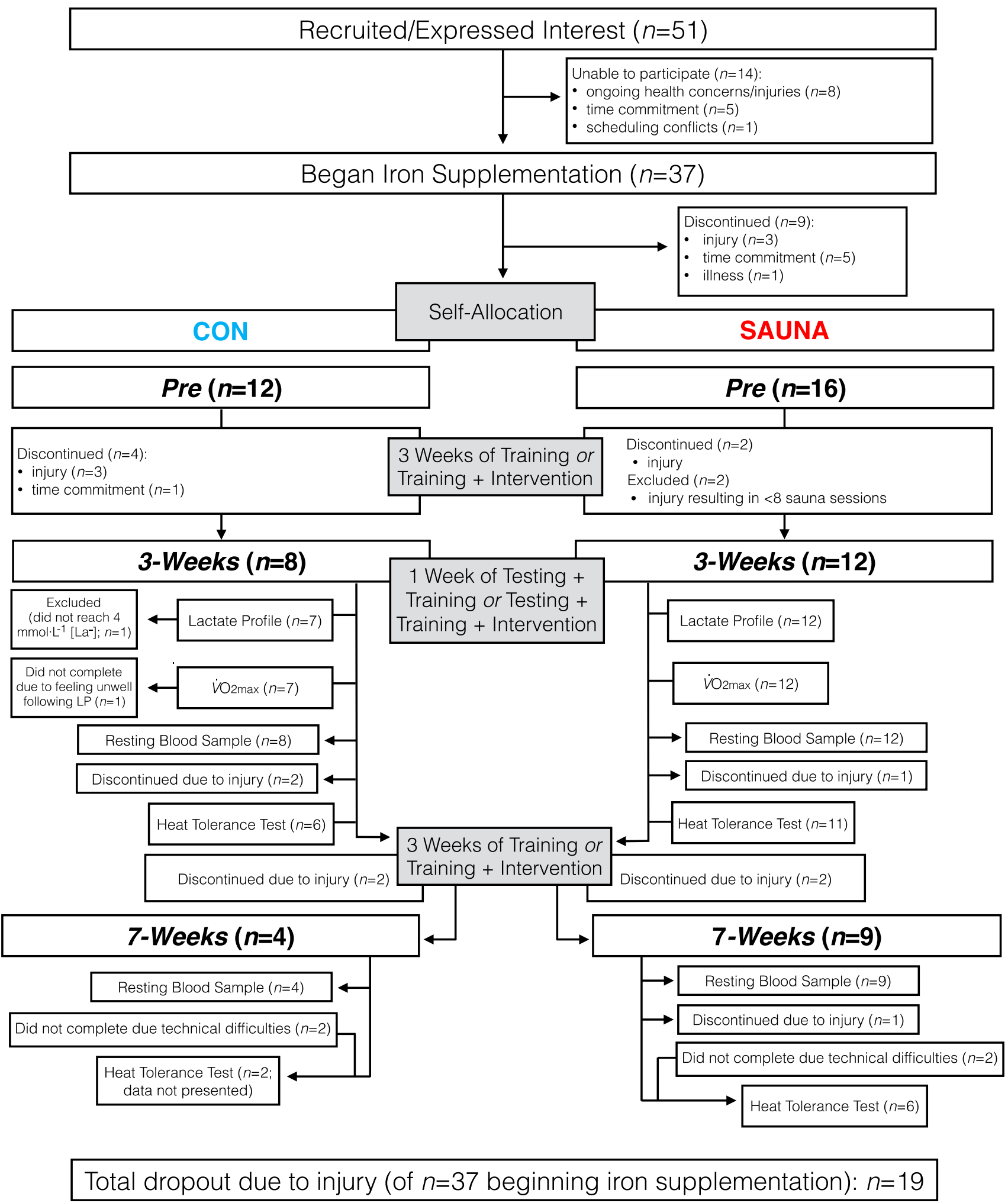

Fig. 2 Progression of participants through phases of the study. $L P$ lactate profile test, $\left[\mathrm{La}^{-}\right]$blood lactate concentration; $V O_{2 m a x}$ maximal aerobic capacity

food they had eaten prior to experimental tests, and to repeat the same diet when re-tested. Participants voided their bladder upon arrival to the laboratory to provide a urine sample, which was analysed for osmolality. If urine osmolality was $\geq 700 \mathrm{mOsm} \mathrm{kg}^{-1}$ (Osmocheck, Vitech Scientific Ltd., West Sussex, United Kingdom; Sawka et al. 2007), 
participants drank $250 \mathrm{~mL}$ of water and did not begin exercising for at least $20 \mathrm{~min}$.

\section{Running heat tolerance test (HTT)}

The running heat tolerance test (HTT) was performed in hot conditions $\left(40{ }^{\circ} \mathrm{C}, 40 \% \mathrm{RH}\right)$ in an environmental chamber (TIS Services, Hampshire, United Kingdom) with a fan-generated airflow of $\sim 4 \mathrm{~m} \mathrm{~s}^{-1}$. Towel-dried, nude body mass was recorded to $0.1 \mathrm{~kg}$ using digital scales (Seca 877, Seca, Hamburg, Germany) before and immediately after the HTT to estimate whole-body sweat loss. Participants wore socks, shoes, and either shorts (males) or shorts and a sports bra (females). Participants ran on a treadmill (H/P/Cosmos Quasar 4.0, H/P/Cosmos, Germany) at $9 \mathrm{~km} \mathrm{~h}^{-1}$ and $2 \%$ gradient for $30 \mathrm{~min}$ (Mee et al. 2015). Rectal temperature $\left(T_{\text {rec }}\right)$, skin temperature $\left(T_{\text {sk }}\right)$, and HR were measured continuously. Perceptual measures, which included ratings of perceived exertion (RPE), thermal comfort and thermal sensation, were obtained in the final min of the HTT. Sweat gland activity of the forearm and upper back were recorded immediately following the HTT. Drinking was not permitted during the test.

\section{Lactate profile test}

The lactate profile test was performed in temperate conditions $\left(\sim 18{ }^{\circ} \mathrm{C}\right)$. Participants completed a light 10 -min warmup before commencing the step-style incremental test on the treadmill. Participants completed 3-min stages with 30-s stops between stages when capillary $\left[\mathrm{La}^{-}\right]$was measured. The test began at $1 \%$ gradient (Jones and Doust 1996) and a speed $4 \mathrm{~km} \mathrm{~h}^{-1}$ slower than a recent $5-\mathrm{km}$ race pace, and increased by $1 \mathrm{~km} \mathrm{~h}^{-1}$ for each stage thereafter. HR and respiratory gas exchange were continuously measured. The test was terminated when $\left[\mathrm{La}^{-}\right]$exceeded $4 \mathrm{mmol} \mathrm{L}^{-1}$, which occurred following 4-7 stages.

\section{Maximal aerobic capacity $\left(\mathrm{VO}_{2 \max }\right)$}

Approximately $10 \mathrm{~min}$ after completing the lactate profile test, participants performed a ramp-style $V \mathrm{O}_{2 \max }$ test. The test began at $1 \%$ gradient and at the speed $2 \mathrm{~km} \mathrm{~h}^{-1}$ slower than the speed at which the participant had surpassed $4 \mathrm{mmol} \mathrm{L}^{-1}\left[\mathrm{La}^{-}\right]$. Speed increased $1 \mathrm{~km} \mathrm{~h}^{-1}$ each minute for the next $2 \mathrm{~min}$, when the speed at which $>4 \mathrm{mmol} \mathrm{L}^{-1}\left[\mathrm{La}^{-}\right]$ had been reached. At this point, the gradient was increased by $1 \%$ each minute until volitional exhaustion. Participants were given consistent and loud encouragement during the test. When participants were re-tested at 3-Weeks, the starting speed and progression of the protocol was repeated to allow for measurement of time-to-exhaustion (TTE). HR and respiratory gas exchange were continuously measured.
Capillary $\left[\mathrm{La}^{-}\right]$was measured $5 \mathrm{~min}$ from the time of exhaustion.

\section{Resting blood sampling and analysis}

On a separate visit (between the hours of 9:00-11:00 h), participants rested in a supine position for a minimum of $10 \mathrm{~min}$, at which time resting HR was assessed and venous blood was drawn from the antecubital vein into a K2EDTAcoated vacutainer for measurement of plasma VEGF and an uncoated vacutainer for measurement of serum EPO. Vacutainers were kept on ice (plasma) or at room temperature (serum) for $45 \mathrm{~min}$ before centrifugation $\left(4^{\circ} \mathrm{C}, 10,000 \mathrm{rpm}\right.$, $10 \mathrm{~min}$ ) (Heraeus Multifuge X1R, Thermo Scientific, Loughborough, UK) and were frozen at $-80^{\circ} \mathrm{C}$ until analysis. Plasma VEGF (Human VEGF DuoSet ELISA; R\&D Systems, Abingdon, UK) and serum EPO (Human Erythropoietin DuoSet ELISA; R\&D Systems, Abingdon, UK) were determined with ELISA kits.

\section{Measures}

$T_{\text {rec }}$ was measured using a rectal thermistor inserted $10 \mathrm{~cm}$ past the anal sphincter (Mon-a-Therm, Covidien, Mansfield, MA, United States). Weighted mean skin temperature was recorded using skin thermistors (Squirrel Thermal Couples, Grant Instruments, Cambridge, UK) attached to four sites on the left side of the body: pectoralis major $\left(T_{\text {chest }}\right)$, biceps brachii $\left(T_{\text {arm }}\right)$, rectus femoris $\left(T_{\text {thigh }}\right)$, and gastrocnemius lateral head $\left(T_{\text {lowerleg }}\right)$. Skin and rectal temperatures were continuously logged at 30-s intervals (Squirrel 2020 series, Eltek, Ltd., United Kingdom). HR (Polar Electro, Kempele, Finland) was recorded continuously (sampling rate of $1 \mathrm{~Hz}$ ) on the Polar Beat application (Polar Beat, Kempele, Finland).

Active sweat glands were quantified using a modifiediodine paper technique with blinded computer aided analysis (Gagnon et al. 2012). Samples were collected by the same researcher from the dorsal side of the thickest segment of the forearm and mid-scapula on the upper back. Training session RPE was measured using a 1-10 point scale $\left(\mathrm{RPE}_{1-10}\right.$; 'very light session' to 'max effort session') and RPE during HTTs was measured using the 6-20 point Borg Scale $\left(\mathrm{RPE}_{6-20}\right.$; Borg 1982). Thermal sensation and thermal comfort were measured using modified 13-point and 10-point scales, respectively (Gagge et al. 1967). Measures of $\left[\mathrm{La}^{-}\right]$ were taken from a fingertip capillary sample and immediately analysed using a Biosen C-Line Lactate analyser (EKF Diagnostics, Penarth, UK), which was quality checked each day and calibrated every $60 \mathrm{~min}$. Respiratory gas exchange was sampled breath-by-breath using open-circuit spirometry (Vyntus CPX, Jaeger, Wuerzberg, Germany). 


\section{Data analysis}

Peak $T_{\text {rec }}$ was the highest $T_{\text {rec }}$ value measured during the HTT. $T_{\text {recRISE }}$ was calculated as the change in $T_{\text {rec }}$ from 0-30 min during the HTT. $T_{\text {sk }}$ was calculated as a weighted average according to Ramanathan (1964):

$T_{\text {sk }}=0.3 \times\left(T_{\text {chest }}+T_{\text {arm }}\right)+0.2 \times\left(T_{\text {thigh }}+T_{\text {lowerleg }}\right)$

Estimated sweat loss was calculated as the difference between Pre- and post-HTT nude body mass. Running speed at $4 \mathrm{mmol} \mathrm{L}^{-1}\left[\mathrm{La}^{-}\right]$was determined using a custom Matlab script (The Mathworks Inc, Natick, USA) to fit a third-order polynomial curve to each individual dataset and interpolate the running speed at $4 \mathrm{mmol} \mathrm{L}^{-1}$. Respiratory gas exchange data were exported as 5-s values and used to calculate oxygen consumption, running economy and respiratory exchange ratio (RER) during the lactate profile and $V \mathrm{O}_{2 \max }$ tests. Running economy $\left(\mathrm{mL} \mathrm{kg}^{-1} \mathrm{~km}^{-1}\right)$ was calculated as oxygen consumption $\left(\mathrm{mL} \mathrm{kg}^{-1} \mathrm{~min}^{-1}\right)$ divided by treadmill speed $\left(\mathrm{km} \mathrm{h}^{-1}\right)$ (Barnes and Kilding 2015). During the lactate profile test, submaximal RER, running economy and HR data were averaged across the final minute of the stage where participants exceeded $4 \mathrm{mmol} \mathrm{L}^{-1}\left[\mathrm{La}^{-}\right]$at baseline, with the same running speed compared at 3-Weeks. $V \mathrm{O}_{2 \max }$ was calculated as the highest rolling 30-s average attained during the test. Successful attainment of $V \mathrm{O}_{2 \max }$ required meeting two of the following three criteria: (1) $\left[\mathrm{La}^{-}\right] \geq 8 \mathrm{mmol} \mathrm{L}^{-1}$ (Howley et al. 1995), (2) RER $\geq 1.10$ (Edvardsen et al. 2014), (3) maximal HR $\geq 90 \%$ of age-predicted maximal HR (220 - age). Additionally, a plateau was confirmed both visually and systematically by a 5 -s average value $\geq 2$ standard deviations lower than the linear forecast of $V \mathrm{O}_{2}$ increase. For individual $T_{\mathrm{sk}}$ sites, if a time-point was lost, the missing data were modelled according to the slope/pattern of other data points from that participant during the previous test. Data at multiple $T_{\mathrm{sk}}$ sites were lost for two SAUNA group participants in the HTTs at 7-Weeks, and therefore SAUNA $T_{\mathrm{sk}}$ at 7-Weeks $(n=4)$ was deemed incomplete and not reported herein. Training data and resting HR were not collected in $n=1$ SAUNA participant for both 3- and 7-Weeks.

\section{Statistical approach}

All data were analysed using SPSS statistical software (SPSS version 25.0.0, SPSS, Chicago, IL, United States). To address the main aims, physiological data from HTT, lactate profile and $V \mathrm{O}_{2 \max }$ tests were compared between SAUNA and CON groups using a one-way analysis of co-variance (ANCOVA), with change scores ( $\Delta$; difference between Pre and 3-Weeks) entered as the dependent variable and Pre-intervention absolute data entered as a co-variate. This allowed all changes to be covariate-adjusted for the baseline values (Vickers and Altman 2001) and yielded a single $p$ value to represent the between-group comparison. This $p$ value reflects the effect of the intervention. Because sex affects cardiorespiratory fitness, sex as a variable was tested for violations related to collinearity and entered as co-variate in analyses of $V \mathrm{O}_{2 \max }$ and running speed at $4 \mathrm{mmol} \mathrm{L}^{-1}$ $\left[\mathrm{La}^{-}\right]$. Effect sizes derived from between-group comparisons are presented as Cohen's $d$, whereby $d>0.8$ are considered large, $0.5-0.8$ moderate and $0.2-0.5$ small. Normality of these data was assessed using Levene's test of equality of error variances, which was not violated on any occasion.

To address the subsidiary aim, a one-way repeated-measures analysis of variance (ANOVA) was used to compare HTT physiological data from the SAUNA group participants at Pre, 3-Weeks and 7-Weeks (time [3 levels]).

Total training frequency and average weekly running distance were compared either between groups (3-Weeks) using an independent $t$ test (group [2 levels]) or within-group (7-Weeks) using a one-way repeated-measures ANOVA (week [7 levels]). Average weekly frequency of each type of training session (i.e., easy run, tempo training, interval training, and long run) were compared using a two-way ANOVA (3-Weeks: between groups, type [4 levels] $\times$ group [2 levels]; 7-Weeks: repeated-measures, type [4 levels] $\times$ week [7 levels]).

Normality of data analysed by ANOVA was assessed using Mauchly's test of sphericity, and Greenhouse-Geisser corrections were applied where assumptions of sphericity were violated. When a significant main effect was found, Dunn-Bonferroni-corrected post hoc comparisons were made. Main effect sizes for ANOVAs were calculated using partial eta-squared $\left(\eta_{p}{ }^{2}\right)$, whereby $\eta_{p}^{2}>0.14$ are considered large, 0.06-0.14 moderate and $<0.06$ small (Cohen 1988).

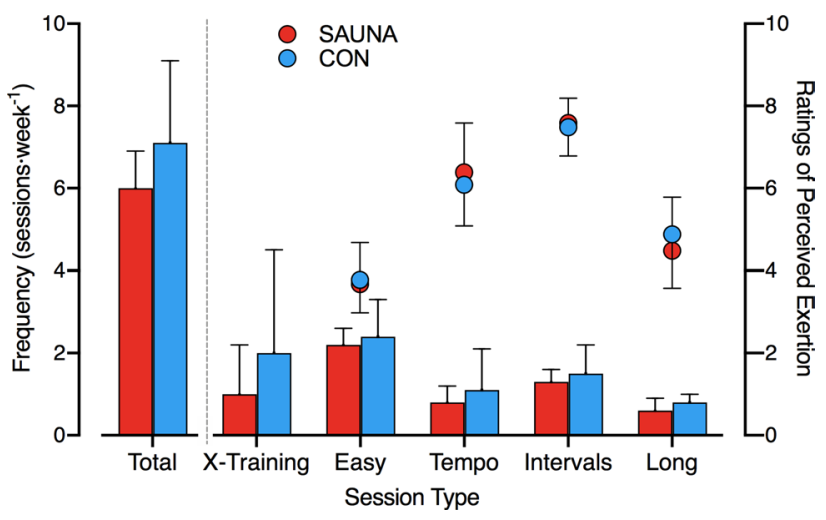

Fig. 3 Training frequency, type (bars, left axis) and session perceived exertion (10-point scale; circles, right axis) for three-weeks training in the post-exercise sauna intervention (SAUNA, red) and control (CON, blue) groups. X-training, cross-training. Data are presented as mean $\pm \mathrm{SD}$ 
Ordinal data, including average running session $\mathrm{RPE}_{1-10}$, and peak $\mathrm{RPE}_{6-20}$, thermal comfort and thermal sensation during HTTs, were compared between groups (3-Weeks) using a Mann-Whitney $U$ test or within-group (7-Weeks) using a Friedman's test with post hoc analysis by Wilcoxon sign-rank tests.

Absolute data and within-subject changes are expressed as mean \pm standard deviation (SD), and $95 \%$ confidence limits ([95\% CL: lower limit, upper limit]) are presented with mean between-group differences. Significance was set at $p<0.05$ for each analysis.

\section{Results}

\section{Training}

3-Weeks: Training sessions were performed $7 \pm 2$ times per week (SAUNA: $6 \pm 1$; CON: $7 \pm 2$ ) in the weeks between Pre and 3-Weeks testing. Average weekly running distances were $53.3 \pm 23.3 \mathrm{~km}$ and $54.5 \pm 28.2 \mathrm{~km}$ in SAUNA and CON, respectively. Training was not different in type, frequency, running distance or perceived exertion between the SAUNA and CON groups at 3-Weeks (all $p>0.05$; Fig. 3).

7-Weeks: Participants in the SAUNA group trained $6 \pm 1$ times per week, and ran $59.1 \pm 21.8 \mathrm{~km} \mathrm{week}^{-1}$. Training was not different in type, frequency, running distance or perceived exertion across the 7 weeks prior to 7-Weeks testing in the SAUNA group (all $p>0.05$ ).

\section{Post-exercise sauna bathing sessions}

3-Weeks: Participants in the SAUNA group attended sauna sessions $3 \pm 1$ times per week, accumulating $9 \pm 1$ sauna sessions before completing the lactate profile and $V \mathrm{O}_{2 \max }$ tests, and $10 \pm 1$ sauna sessions before completing the HTT. Participants remained in the sauna for $28 \pm 2$ min each session, totalling $290 \pm 48$ min of sauna exposure. Peak HR reached $127 \pm 10$ beats $\mathrm{min}^{-1}$ whilst in the sauna.

7-Weeks: Participants in the SAUNA group attended sauna sessions $3 \pm 1$ times per week, accumulating $19 \pm 1$ sauna sessions before completing the final HTT at 7-Weeks. These participants remained in the sauna for $28 \pm 1$ min each session, totalling $528 \pm 29 \mathrm{~min}$ of sauna exposure. Peak HR reached $120 \pm 14$ beats $\min ^{-1}$ whilst in the sauna. Frequency, duration, and peak HR were not different between the sauna sessions completed before 3-Weeks testing as compared to those completed between 3-Weeks and 7-Weeks testing (all $p>0.05$ ).

\section{Menstrual cycle}

Female participants completed all experimental trials at Pre, 3-Weeks and 7-Weeks $28 \pm 2$ days apart. Normally menstruating participants $(n=8)$ reported menstrual cycles ranging between 26-31 days and five completed all tests in the same phase of their menstrual cycle. Of the remaining three, one SAUNA participant completed all Pre tests in the follicular phase and all 3-Weeks tests in the luteal phase, one SAUNA participant completed temperate exercise tests at Pre in the luteal phase and at 3-Weeks in the follicular phase (though HTTs were completed in the same phase), one CON participant completed temperate exercise tests at Pre in the follicular phase and at 3-Weeks in the luteal phase (HTTs completed in the same phase). Participants using oral contraceptives $(n=2)$ completed all tests in the active pilltaking phase.

\section{Resting measures}

3-Weeks: Changes in resting HR were not different between groups at 3 -Weeks as compared to Pre (SAUNA: $58 \pm 6$ vs $55 \pm 9$ beats $\mathrm{min}^{-1}$; CON: $54 \pm 6$ vs $51 \pm 4$ beats $\mathrm{min}^{-1}$, at Pre vs 3 -Weeks, respectively; $p=0.90, d=0.06$ ). Changes in body mass were not different between groups (SAUNA: $61.0 \pm 6.3$ vs $61.2 \pm 6.4 \mathrm{~kg}$; CON: $63.8 \pm 5.5$ vs $63.5 \pm 5.4 \mathrm{~kg}$, at Pre vp 3-Weeks, respectively; $p=0.43, d=0.45)$. Serum EPO was detectable in 17 out of 20 samples (SAUNA, $n=12$; CON, $n=5)$. Changes in resting serum EPO were not different $(p=0.82, d=0.24)$ between groups (SAUNA: $3.8 \pm 2.7$ vs $3.1 \pm 1.6 \mathrm{mIU} \mathrm{mL}^{-1}$; CON: $2.8 \pm 2.3$ vs $2.6 \pm 1.9$

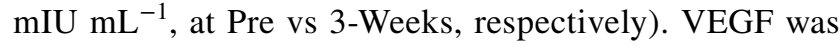
detectable in 10 out of 20 samples (SAUNA, $n=5$; CON, $n=5)$. Changes in resting plasma VEGF were not different ( $p=0.09, d=0.35$ ) between groups (SAUNA: $128.5 \pm 121.9$ vs $117.9 \pm 106.4 \mathrm{pg} \mathrm{mL}^{-1}$; CON: $183.0 \pm 234.4$ vs $179.8 \pm 274.7 \mathrm{pg} \mathrm{mL}^{-1}$, at Pre vs 3-Weeks, respectively).

7-Weeks: Participants in the SAUNA group showed a trend $(p=0.06)$ for a decrease in resting HR across time, though this was not significant $(57 \pm 6,53 \pm 10$ and $53 \pm 7$ beats $\min ^{-1}$ at Pre, 3-Weeks and 7-Weeks, respectively). Serum EPO did not significantly change across time (3.6 $\pm 1.6,3.1 \pm 1.8$ and $4.4 \pm 2.8 \mathrm{mIU} \mathrm{mL}^{-1}$ at Pre, 3-Weeks and 7 -Weeks, respectively; $p=0.18$ ). VEGF was detectable in $n=3$ samples and therefore not statistically compared $\left(77.9 \pm 55.6,77.5 \pm 39.4\right.$ and $76.1 \pm 51.6 \mathrm{pg} \mathrm{mL}^{-1}$ at Pre, 3-Weeks and 7-Weeks, respectively). 
Table 1 Physiological and perceptual responses during the running Heat Tolerance Test

\begin{tabular}{|c|c|c|c|c|}
\hline & \multicolumn{2}{|l|}{ SAUNA } & \multicolumn{2}{|l|}{$\mathrm{CON}$} \\
\hline & Pre & 3-Weeks & Pre & 3-Weeks \\
\hline Peak $T_{\text {rec }}\left({ }^{\circ} \mathrm{C}\right)$ & $38.6 \pm 0.5$ & $38.3 \pm 0.3 *$ & $38.6 \pm 0.4$ & $38.5 \pm 0.3$ \\
\hline$T_{\text {recRISE }}\left({ }^{\circ} \mathrm{C}\right)$ & $1.4 \pm 0.4$ & $1.2 \pm 0.3$ & $1.5 \pm 0.5$ & $1.5 \pm 0.5$ \\
\hline Peak $T_{\text {sk }}\left({ }^{\circ} \mathrm{C}\right)$ & $36.4 \pm 1.3$ & $35.7 \pm 0.8^{*}$ & $36.3 \pm 1.4$ & $36.5 \pm 1.2$ \\
\hline Peak HR (beats $\min ^{-1}$ ) & $163 \pm 24$ & $152 \pm 19^{*}$ & $157 \pm 16$ & $158 \pm 21$ \\
\hline Sweat loss (kg) & $0.8 \pm 0.2$ & $0.8 \pm 0.2 *$ & $0.8 \pm 0.3$ & $0.7 \pm 0.1$ \\
\hline Forearm active sweat glands (per $\mathrm{cm}^{2}$ ) & $51 \pm 24$ & $70 \pm 20 *$ & $45 \pm 20$ & $44 \pm 24$ \\
\hline Upper back active sweat glands (per $\mathrm{cm}^{2}$ ) & $64 \pm 15$ & $70 \pm 24$ & $71 \pm 16$ & $63 \pm 26$ \\
\hline Peak $\mathrm{RPE}_{6-20}$ & $12 \pm 2$ & $10 \pm 2$ & $11 \pm 2$ & $11 \pm 2$ \\
\hline Peak thermal sensation & $10 \pm 1$ & $9 \pm 1^{*}$ & $9 \pm 1$ & $9 \pm 0$ \\
\hline Peak thermal comfort & $5 \pm 2$ & $4 \pm 2 *$ & $3 \pm 2$ & $4 \pm 2$ \\
\hline
\end{tabular}

Data are presented as mean $\pm \mathrm{SD}$

$H R$ heart rate, $T_{r e c}$ rectal temperature, $T_{\text {recRISE }}$ change in rectal temperature during exercise, $R P E_{6-20}$ rating of perceived exertion (6-20 point scale). Thermal sensation and thermal comfort were measured on 13- and 10-point scales, respectively

*Change from Pre to 3-Weeks in SAUNA group significantly different from CON $(p<0.05)$

\section{Running heat tolerance test}

\section{Body temperatures}

3-Weeks: Body temperature responses at Pre and 3-Weeks are detailed in Table 1. The SAUNA group exhibited a $0.2{ }^{\circ} \mathrm{C}[0.0,0.4]$ greater reduction in peak $T_{\text {rec }}$ than the CON group at 3 -Weeks as compared to Pre ( $p=0.04, d=0.67$; Fig. 4a). However, changes in $T_{\text {recRISE }}$ were not different between groups $(p=0.14 ; d=0.54$; Fig. $4 \mathrm{~b})$. The SAUNA group exhibited a $0.8^{\circ} \mathrm{C}[0.1,1.5]$ greater reduction in peak $T_{\text {sk }}$ than the CON group at 3-Weeks as compared to Pre $(p=0.03, d=0.99)$.

7-Weeks: Peak $T_{\text {rec }}$ in the SAUNA group showed a large and significant main effect of time $\left(p<0.01, \eta_{p}{ }^{2}=0.84\right.$; Fig. 4d). Post hoc analysis revealed that peak $T_{\text {rec }}$ was $0.3{ }^{\circ} \mathrm{C}[0.0,0.6]$ lower in SAUNA at 3-Weeks $(p=0.04)$ and $0.4{ }^{\circ} \mathrm{C}[0.2,0.6]$ lower at 7 -Weeks $(p<0.01)$, as compared to Pre. Furthermore, peak $T_{\text {rec }}$ was $0.1[0.0,0.3]{ }^{\circ} \mathrm{C}$ lower at 7-Weeks as compared to 3-Weeks $(p=0.03)$.

\section{Heart rate}

3-Weeks: The SAUNA group exhibited an 11 beats $\min ^{-1}$ $[0,22]$ greater reduction in peak HR during the HTT than the CON group at 3 -Weeks as compared to Pre $(p<0.05$, $d=1.16$; Fig. 4c; Table 1).

7-Weeks: Peak HR in the SAUNA group showed a large and significant main effect of time $\left(p=0.02, \eta_{p}{ }^{2}=0.54\right.$; Fig. 4f). Post hoc analysis showed no significant differences, however there was a trend for peak HR to be lower at 3 -Weeks as compared to Pre $\left(-11\right.$ beats $\min ^{-1}[+2,-23]$; $p=0.08)$.

\section{Sweating}

3-Weeks: Absolute sweating data at Pre and 3-Weeks are detailed in Table 1 . The CON group showed an attenuated sweat loss of $0.2 \mathrm{~kg}[0.0,0.3]$ during the HTT, as compared to the SAUNA group at 3-Weeks vs Pre $(p=0.02, d=0.95)$. The SAUNA group exhibited a $54 \pm 59 \%$ increase in sweat gland activity on the forearm, equating to a 22 active glands per $\mathrm{cm}^{2}$ (Cohen 1988; Racinais et al. 2015b) greater increase than the CON group's $6 \pm 17 \%$ reduction, at 3 -Weeks as compared to Pre $(p<0.01, d=1.40)$. Changes in sweat gland activity on the upper back were not different between groups $(p=0.59, d=0.35)$.

7-Weeks: Sweat loss in the SAUNA group was not different between any of the HTTs $(0.9 \pm 0.2,0.8 \pm 0.1$ and $0.8 \pm 0.2 \mathrm{~kg}$ at at Pre, 3-Weeks and 7-Weeks, respectively; $\left.p=0.21, \eta_{p}{ }^{2}=0.27\right)$. Sweat gland activity on the forearm showed a large and significant main effect of time (48 \pm 17 , $66 \pm 14$ and $69 \pm 13$ active glands per $\mathrm{cm}^{2}$ at Pre, 3-Weeks and 7-Weeks, respectively; $p=0.01, \eta_{p}{ }^{2}=0.57$ ). Post hoc analysis showed no significant differences, however there was a trend for sweat gland activity on the forearm to be greater at 3-Weeks as compared to Pre $(p=0.07)$. Sweat gland activity on the upper back was not different between any of the HTTs $(72 \pm 6,77 \pm 20$ and $75 \pm 7$ active glands per $\mathrm{cm}^{2}$ at Pre, 3-Weeks and 7-Weeks, respectively; $p=0.33$, $\left.\eta_{p}^{2}=0.20\right)$.

\section{Perceptual measures}

3-Weeks: Perceptual data at Pre and 3-Weeks are detailed in Table 1 . The SAUNA group exhibited a $1 \pm 1$ scale point greater reduction in peak thermal sensation $(p=0.02)$ and a 
Fig. 4 Delta $(\Delta)$ peak rectal temperature $\left(T_{\text {rec }} ; \mathbf{a}\right)$, rise in rectal temperature $\left(T_{\text {recRISE }}\right.$; b) and peak heart rate (HR; c) during the running heat tolerance test (HTT; $40{ }^{\circ} \mathrm{C}$, $40 \% \mathrm{RH}$ ) in the SAUNA (red) and CON (blue) groups from baseline (Pre) to following three-weeks post-exercise sauna bathing intervention or control (3-Weeks). Absolute peak Trec (d), TrecRISE (e) and peak heart rate (f) during the HTT at Pre, 3-Weeks and following seven-weeks intervention (7-Weeks). Horizontal black lines $(\mathbf{a}-\mathbf{c})$ or bars $(\mathbf{d}-\mathbf{f})$ and error bars represent the group mean \pm SD. Circles represent individual participant data. The participant who completed both SAUNA and CON interventions is indicated by an $X$. *Significant difference in SAUNA vs $\mathrm{CON}$; ${ }^{\text {significant main effect }}$ of time; ${ }^{\text {asignificant difference }}$ from Pre; ${ }^{b}$ significant difference from 3-Weeks, $(p<0.05)$
Table 2 Physiological responses to temperate exercise tests
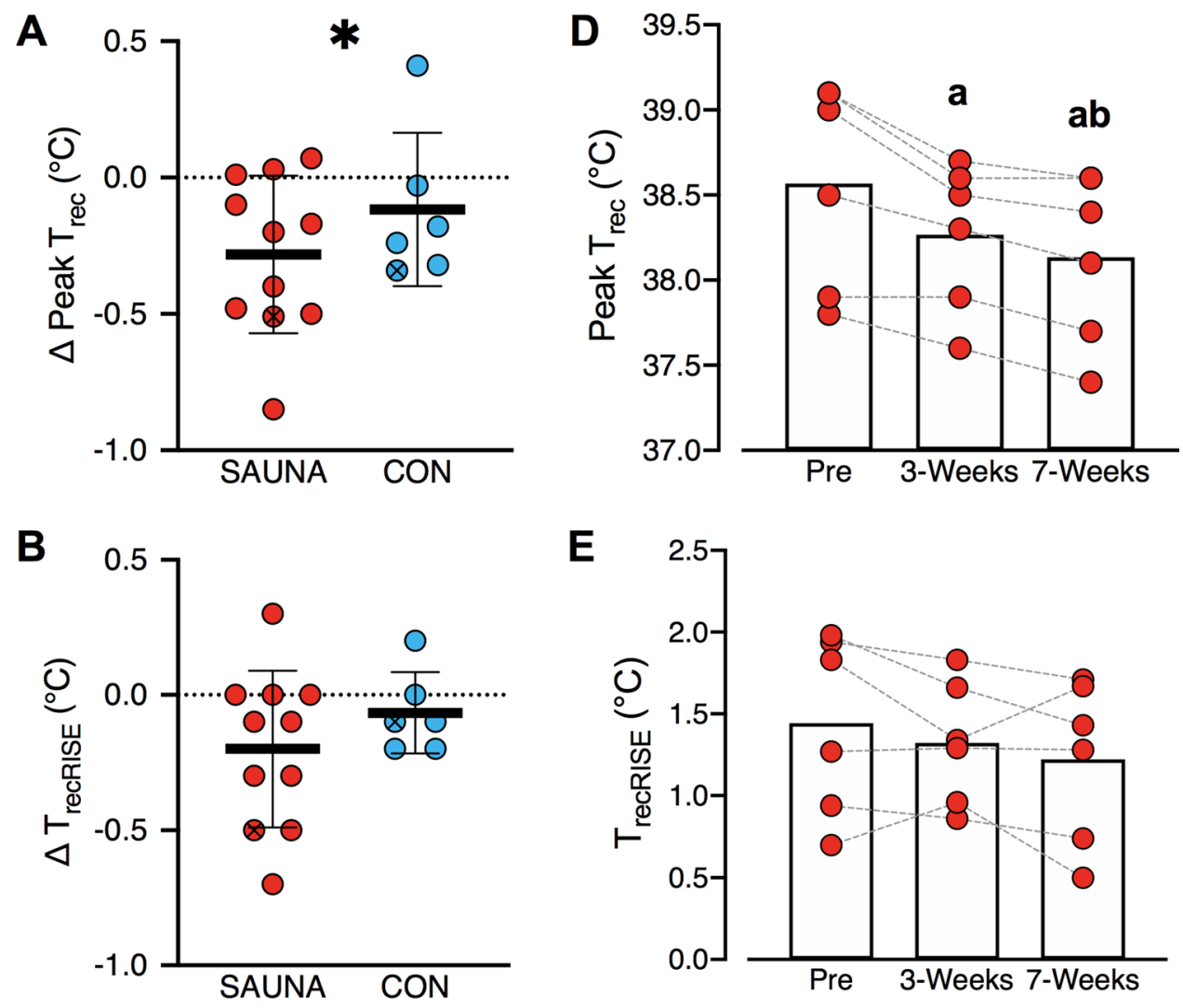

E
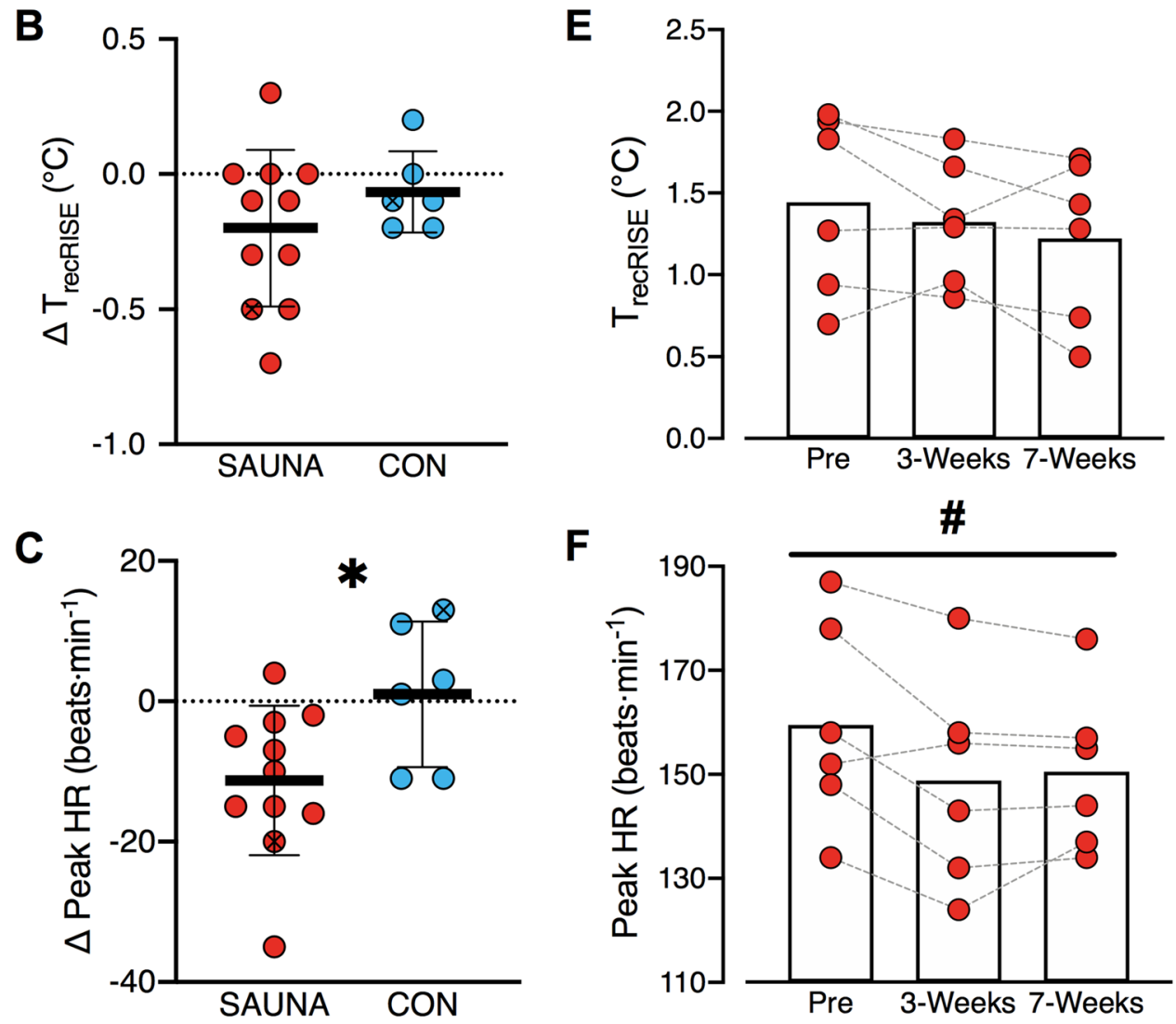

\begin{tabular}{|c|c|c|c|c|}
\hline & \multicolumn{2}{|l|}{ SAUNA } & \multicolumn{2}{|l|}{$\mathrm{CON}$} \\
\hline & Pre & 3-Weeks & Pre & 3-Weeks \\
\hline$V \mathrm{O}_{2 \max }\left(\mathrm{L} \mathrm{min}^{-1}\right)$ & $3.42 \pm 0.81$ & $3.65 \pm 0.75^{*}$ & $3.72 \pm 0.76$ & $3.73 \pm 0.55$ \\
\hline TTE (s) & $426 \pm 57$ & $474 \pm 59 *$ & $439 \pm 62$ & $433 \pm 79$ \\
\hline Running speed at $4 \mathrm{mmol} \mathrm{L}^{-1}\left[\mathrm{La}^{-}\right]\left(\mathrm{km} \mathrm{h}^{-1}\right)$ & $15.6 \pm 1.7$ & $16.2 \pm 1.7 *$ & $16.8 \pm 1.5$ & $16.8 \pm 1.4$ \\
\hline Submaximal HR (beats $\min ^{-1}$ ) & $188 \pm 9$ & $183 \pm 8$ & $178 \pm 12$ & $182 \pm 12$ \\
\hline Submaximal RER & $1.01 \pm 0.06$ & $1.00 \pm 0.05$ & $1.02 \pm 0.03$ & $1.04 \pm 0.03$ \\
\hline Submaximal RE $\left(\mathrm{mL} \mathrm{kg}^{-1} \min ^{-1}\right)$ & $189 \pm 14$ & $195 \pm 11$ & $187 \pm 25$ & $194 \pm 22$ \\
\hline
\end{tabular}

Data are presented as mean $\pm \mathrm{SD}$

$V O_{2 \max }$ maximal aerobic capacity, TTE time to exhaustion, $\left[\mathrm{La}^{-}\right]$blood lactate concentration, $H R$ heart rate, $R E R$ respiratory exchange ratio, $R E$ running economy

$*$ Change from Pre to 3-Weeks in SAUNA group significantly different from CON $(p<0.05)$ 

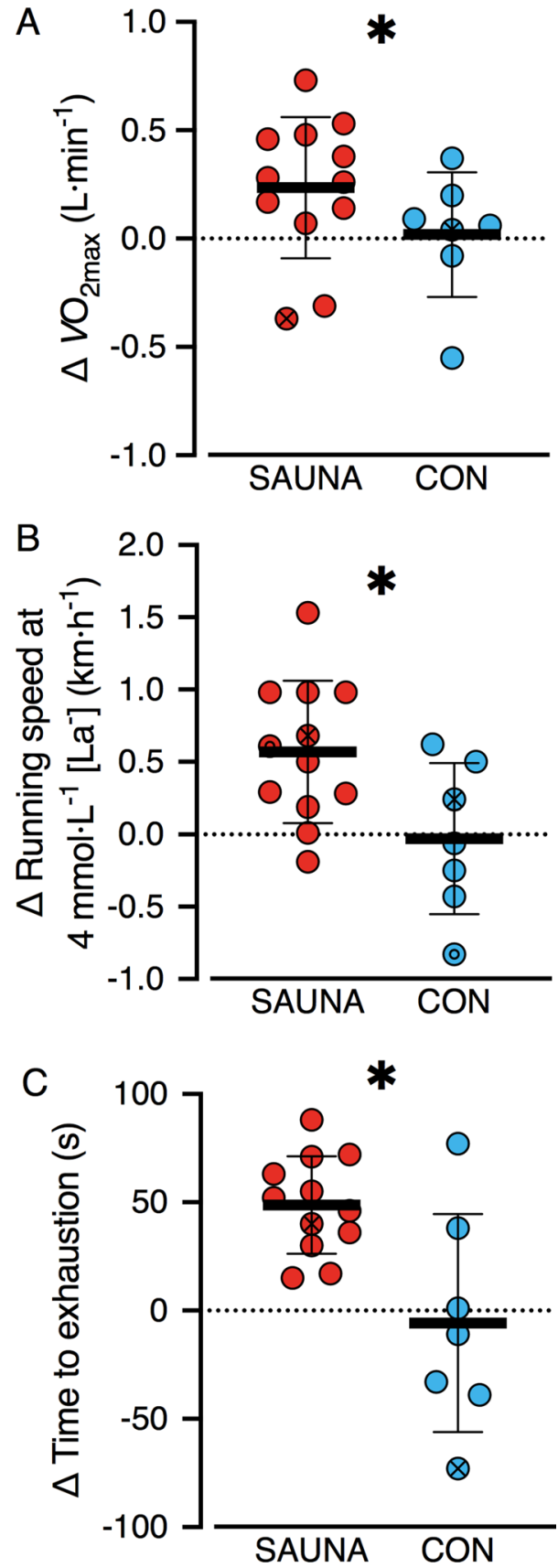

Fig. 5 "Delta $(\Delta)$ exercise performance markers in temperate conditions $\left(18{ }^{\circ} \mathrm{C}\right)$ at baseline (Pre) to following three-weeks (3-Weeks) post-exercise sauna bathing intervention (SAUNA; red, left panel) or control (CON; blue, right panel). Mean \pm SD (horizontal lines and error bars) plus individual participant data (circles) presented for delta $(\Delta)$ maximal aerobic capacity $\left(V \mathrm{O}_{2 \max }, \mathbf{a}\right)$, running speed at $4 \mathrm{mmol} \mathrm{L}{ }^{-1}$ blood lactate concentration $\left(\left[\mathrm{La}^{-}\right]\right)(\mathbf{b})$ and running time to exhaustion (c). The two participants who completed experimental tests following both SAUNA and CON interventions as indicated by an $X$ and (in $\mathbf{b}$ only) an $O$. *Significant difference in SAUNA vs CON $(p<0.05)$
$2 \pm 2$ scale point greater reduction in peak thermal comfort $(p<0.01)$ during the HTT than the CON group at 3-Weeks as compared to Pre. There was also a trend $(p=0.08)$ for a $2 \pm 2$ scale point greater reduction in $\mathrm{RPE}_{6-20}$ during the HTT in the SAUNA group than the CON group at 3-Weeks as compared to Pre.

7-Weeks: Peak RPE in the SAUNA group was significantly different between the HTTs (main effect: $p=0.04$; $12 \pm 3,11 \pm 2$ and $10 \pm 2$ at Pre, 3-Weeks and 7-Weeks, respectively). Post hoc analysis revealed peak RPE was lower at 7-Weeks as compared to Pre $(p=0.04)$ and as compared to 3 -Weeks $(p=0.03)$. There was a trend $(p=0.08)$ for a reduction in peak RPE at 3-Weeks as compared to Pre. Thermal sensation was not different between HTTs (main effect: $p=0.12 ; 10 \pm 1,9 \pm 1$ and $9 \pm 1$ at Pre, 3 -Weeks and 7-Weeks, respectively). Similarly, thermal comfort was not different between HTTs (main effect: $p=0.09 ; 5 \pm 2,4 \pm 2$ and $3 \pm 1$ at Pre, 3-Weeks and 7-Weeks, respectively).

\section{Lactate profile test}

Physiological responses during the lactate profile test at Pre and 3-Weeks are detailed in Table 2. The SAUNA group exhibited a $4 \pm 3 \%$ improvement in running speed at $4 \mathrm{mmol} \mathrm{L}^{-1}\left[\mathrm{La}^{-}\right]$, equating to a $0.6 \mathrm{~km} \mathrm{~h}^{-1}[0.1,1.0]$ greater improvement than the $\mathrm{CON}$ group $(0 \pm 3 \%)$ at 3 -Weeks as compared to Pre ( $p=0.01, d=1.19$; Fig. $5 \mathrm{~b})$.

During the lactate profile test, there was a trend $(p=0.06$; $d=1.40$ ) for a greater reduction in submaximal HR in the SAUNA group than in the CON group at 3-Weeks as compared to Pre $\left(-7\right.$ beats $\left.\min ^{-1}[0,-14]\right)$. Neither changes in submaximal running economy ( $p=0.73, d=0.27$ ) nor RER $(p=0.14, d=0.30)$ were different between groups.

\section{Maximal aerobic capacity $\left(\mathrm{VO}_{2 \max }\right)$ test}

The SAUNA group exhibited an $8 \pm 12 \%$ improvement in $V \mathrm{O}_{2 \max }$, equating to a $0.27 \mathrm{~L} \mathrm{~min}^{-1}[0.05,0.49]$ greater improvement than the $\mathrm{CON}$ group $(+2 \pm 8 \%)$ at 3 -Weeks as compared to Pre ( $p=0.02, d=0.69$; Fig. 5a; Table 2). Similarly, the SAUNA group exhibited a $12 \pm 6 \%$ improvement in TTE during the $V \mathrm{O}_{2 \max }$ test, equating to a $54 \mathrm{~s}[17,90]$ greater improvement than the $\mathrm{CON}$ group $(-1 \pm 11 \%)$ at 3 -Weeks as compared to Pre ( $p<0.01, d=1.56$; Fig. 5 c; Table 2$)$.

\section{Discussion}

The main findings of this study are that 3 weeks of postexercise sauna bathing induced heat acclimation adaptations (reduced HR, $T_{\text {rec }}$ and $T_{\text {sk }}$, and increased sweat gland 
activity), and improved markers of exercise performance in temperate conditions (i.e., $V \mathrm{O}_{2 \max }$, running speed at $4 \mathrm{mmol} \mathrm{L}{ }^{-1}\left[\mathrm{La}^{-}\right]$and TTE), to a greater extent than endurance training alone. These improvements were consistent with the study hypothesis. Following 7 weeks of post-exercise sauna bathing, $T_{\text {rec }}$ was the only physiological variable measured during the HTT that showed further improvements. However, the additional $0.1{ }^{\circ} \mathrm{C}$ reduction in peak $T_{\text {rec }}$ from 3-Weeks to 7-Weeks may not be physiologically meaningful. Whilst participants did adhere well to the intervention, the dropout rate was high (51\%; mainly due to injuries acquired during normal training in both groups). This drop-out rate was an important finding in-and-of-itself as it highlights the difficulty of implementing a 7-week long heat acclimation protocol in addition to the training programme of competitive endurance runners.

\section{Exercise heat tolerance}

The mean $0.3{ }^{\circ} \mathrm{C}$ reduction in peak $T_{\text {rec }}$ observed in the SAUNA group at 3-Weeks surpasses the coefficient of variation of the HTT $\left(0.34 \%\right.$, corresponding to $0.1{ }^{\circ} \mathrm{C}$ in the current dataset) as validated by Mee and colleagues (2015). Furthermore, the $0.2{ }^{\circ} \mathrm{C}$ difference in the change in peak $T_{\text {rec }}$ between the SAUNA and the CON groups meets the physiologically meaningful threshold identified in a meta-analysis by Tyler and colleagues (2016). This reduction in peak $T_{\text {rec }}$ in the SAUNA group may have resulted from an increased heat dissipation, possibly by means of increased skin blood flow combined with greater peripheral sweat gland activation to improve evaporative heat loss efficiency (Taylor 2014; Smith and Havenith 2019), given we did not observe increased whole-body sweat loss. The $0.8{ }^{\circ} \mathrm{C}$ greater reduction in peak $T_{\mathrm{sk}}$ observed in the SAUNA group at 3-Weeks as compared to the CON group is consistent with other heat acclimation interventions, as Tyler et al. (2016) reported a $0.5 \pm 0.5^{\circ} \mathrm{C}$ reduction in peak $T_{\text {sk }}$ across 67 interventions. Similarly, the 11 beats $\min ^{-1}$ greater reduction in peak HR observed in the SAUNA group at 3-Weeks as compared to the CON group is consistent with other heat acclimation interventions, which report a $16 \pm 6$ beats $\min ^{-1}$ reduction in peak HR across 118 interventions (Tyler et al. 2016). This reduction in peak HR in the SAUNA group was likely mediated by increased plasma and/or blood volume, as was demonstrated using a similar post-exercise sauna bathing protocol by Scoon et al. (2007), though these variables were not measured in the current study. Though endurance training may (Nadel et al. 1974; Ichinose et al. 2009; untrained cohorts, 10 days to 3 months training) or may not (McGarr et al. 2014; moderately-trained cohort, 2 week training) improve thermoregulation during exercise heat stress, the changes in $T_{\mathrm{rec}}, T_{\mathrm{sk}}$ and HR in the SAUNA group were greater than those observed in the CON group.
Overall, this indicates that the improvements observed in the SAUNA group during the HTT were as a result of the postexercise sauna intervention as opposed to normal training. Therefore, intermittent post-exercise sauna bathing appears to induce thermoregulatory and cardiovascular adaptations that are superior to 3-weeks normal training, and of a similar magnitude to those observed in other heat acclimation protocols in the literature (Tyler et al. 2016). These improvements occurred despite using only ten 30-min exposures and did not cause any disruptions to normal training.

Sweat loss during the HTT differed between groups. In the CON group, there was a reduction in sweat loss during the HTT at 3-Weeks. Conversely, there was no change in sweat loss in the SAUNA group during the HTT at 3-Weeks, despite their lower body temperature (i.e., $T_{\text {re }}$ and $T_{\text {skin }}$ ). This indicates a possible seasonal change in sweat rate for the CON group (Matsumoto et al. 1990), whereas the SAUNA group showed an enhanced evaporative heat loss for a given body temperature. Further, at 3-Weeks, the SAUNA group exhibited a substantially greater increase $(+54 \%)$ in sweat gland activation on the forearm than the $\mathrm{CON}$ group $(-6 \%)$. Though sweat gland activation is not indicative of sweat gland output, these findings are supported by a recent study that showed a greater relative sweat output on the limbs following heat acclimation (Smith and Havenith 2019). Notably, the increase in sweat gland activation on the forearm without increases in total sweat loss (as exhibited by the SAUNA group in the current study) may be a more efficient adaptation for athletes competing in hot-humid environments, as it would improve evaporative capacity without exacerbating dehydration (Alber-Wallerström and Holmér 1985).

Besides the further $\sim 0.1{ }^{\circ} \mathrm{C}$ reduction in peak $T_{\text {rec }}$ at 7-Weeks in the SAUNA group, physiological adaptations appeared to plateau by 3-Weeks, despite nearly doubling the participants' total sauna exposures by 7-Weeks. This plateau is consistent with data from active heat acclimation interventions, whereby changes in heart rate and body temperatures are nearly maximised by $\sim 7$ days (Griefahn 1997; Periard et al. 2015). Interestingly, $\mathrm{RPE}_{6-20}$ was only decreased at 7-Weeks. Whether this would coincide with the anticipated steady increase in self-paced exercise capacity in the heat with a long-term intervention (Periard et al. 2015; Racinais et al. 2015b) is unknown, as the HTT was completed at a fixed workload.

\section{Temperate exercise performance}

Our data demonstrate that 3 weeks of intermittent post-exercise sauna bathing is ergogenic for exercise performance in temperate conditions in well-trained participants. The training status of the athletes in the current study make these results especially meaningful. The $8 \%$ mean improvement 
in $V \mathrm{O}_{2 \max }$ observed in the current study ( $\sim 6 \%$ more than the CON group) is comparable to the $5 \%$ observed by Lorenzo and colleagues (2010) when using a 10-day active heat acclimation protocol. Possible mechanisms facilitating this improvement in aerobic capacity with heat acclimation include a greater maximum cardiac output, as demonstrated by Lorenzo et al. (2010) in their study, or increases in total haemoglobin mass, which are possible in an intervention stretching over multiple weeks, as more recently demonstrated by Rønnestad et al. (2020). The iron supplements given to participants in the current study may have helped to facilitate improvements in oxygen carrying capacity, though participants were not given iron supplements by Rønnestad et al. (2020) or by Scoon et al. (2007), who both observed haematological adaptations following heat acclimation.

Three weeks of post-exercise sauna bathing also increased running speed at $4 \mathrm{mmol} \mathrm{L}^{-1}\left[\mathrm{La}^{-}\right]$by $\sim 4$, equating to a $0.6 \mathrm{~km} \mathrm{~h}^{-1}$ greater improvement than in the CON group. These results were similar to the 5\% improvement in cycling power output at $4 \mathrm{mmol} \mathrm{L}^{-1}\left[\mathrm{La}^{-}\right]$reported by Lorenzo et al. (2010), and are supported by other observations of reduced lactate accumulation during exercise following heat acclimation (Young et al. 1985). Although this improvement in running speed at $4 \mathrm{mmol} \mathrm{L}^{-1}\left[\mathrm{La}^{-}\right]$was accompanied by a trend for a greater reduction in submaximal HR in the SAUNA group, running economy was not different during the lactate profile test at 3-Weeks. Thus, changes in $\left[\mathrm{La}^{-}\right]$may have been due to short-term adaptations such as a decreased rate of glycogenolysis (Febbraio et al. 1994; Kirwan et al. 1987), or due to better splanchnic perfusion (and thus improved lactate removal) and haemodilution via increased blood and plasma volume (Scoon et al. 2007; Lorenzo et al. 2010). Alternatively, the multi-week design of the current intervention might have allowed time for heat acclimation-induced mitochondrial biogenesis to manifest (Tamura et al. 2014; Hafen et al. 2018).

The SAUNA group's $\sim 12 \%$ increase in TTE following 3 -Weeks post-exercise sauna bathing was superior to the CON group's $\sim 1 \%$ reduction in TTE. The mean improvement in the SAUNA group was lower than the $32 \%$ increase observed by Scoon et al. (2007), however there were important differences in the TTE tests used, making comparisons difficult. Scoon and colleagues implemented a 15-min TTE test at the runners' best $5-\mathrm{km}$ race pace and $0 \%$ gradient. In the current study, the TTE test increased in either speed or gradient each minute and on average participants reached volitional exhaustion at $\sim 8 \mathrm{~min}$, but at a running speed at which they had reached $4 \mathrm{mmol} \mathrm{L}^{-1}\left[\mathrm{La}^{-}\right]$and at a $4 \%$ incline. Despite using a different style of TTE test, this study builds on evidence for the ergogenic benefits of post-exercise sauna bathing provided by Scoon and colleagues, with the additions of the gold-standard test of aerobic capacity, a robust submaximal exercise test and a larger, mixed-sex cohort.

\section{Training and intervention}

Of the 37 participants who began the experiment, 19 athletes succumbed to an injury (ranging from minor injuries that prevented training for $\sim 1$ week, to more severe injuries such as stress fractures or sprains) and did not complete the study. This is consistent with data reported in a review by van Gent et al. (2007), whereby incidences of lower extremity running injuries ranged between 19 and $79 \%$ in distance runners, with the highest rates observed in athletes reporting weekly running distances and training frequencies similar to those in the current study (i.e., females running 48-63 $\mathrm{km}$ week $^{-1}$, males running $64+\mathrm{km} \mathrm{week}^{-1}$, and athletes training 6-7 days week ${ }^{-1}$ ). For those who completed the intervention, training was consistent across the 7 weeks of post-exercise sauna bathing. Importantly, unlike active heat acclimation protocols that require changes to an athlete's training to accommodate heat acclimation sessions, there were no differences in training between the CON group and SAUNA group in this study. This included similar perceived training intensity and training volume (i.e., frequency and type of sessions and weekly running distance) across the intervention period.

\section{Blood markers}

Serum EPO changes in the SAUNA group were not significantly different from the CON group at 3-Weeks, and did not significantly change in the SAUNA group by 7-Weeks. As EPO is inherently variable in response to environmental stimuli (Chapman 2013; Płoszczyca et al. 2018), additional data may be required to fully understand this effect. Furthermore, serum EPO reflects a balance between renal EPO production and EPO consumption in the bone marrow (Rusko et al. 2004), making it difficult to discern the influence of either production or consumption by measurement of circulating EPO levels alone. Although the subsequent haematological responses of either increased consumption or production of EPO (i.e., increased red blood cell production) would coincide with the improved aerobic capacity observed at 3-Weeks in the current study and the increased red cell volume observed by Scoon et al. (2007), we were unable to measure red blood cell mass in the current study. VEGF was largely undetectable across the study, as a number of participant samples fell outside the ELISA kit's limits of detection for the measurement, providing little insight into any possible angiogenesis with post-exercise sauna bathing. 


\section{Perspectives and considerations}

These results contribute to the growing body of research supporting favourable health and performance outcomes following passive heating interventions. The investigation of the efficacy of post-exercise sauna bathing to induce heat acclimation is especially timely as the Tokyo 2020 Olympic games (though postponed) are predicted to be the hottest Summer Olympic Games to date. We observed that heat acclimation was nearly maximised by three weeks of intermittent post-exercise sauna bathing and 7 weeks did not induce any further meaningful physiological improvements. Thus, athletes need only incorporate a post-exercise sauna regime for 3 weeks to achieve beneficial adaptations.

This study did not exclude participants based on menstrual cycle or hormonal contraceptive use, and instead included a random sample of female athletes in random phases of their menstrual cycle. The diverse menstrual cycle patterns and contraceptive choices observed in this cohort is representative of the female athlete population. Whilst menstrual cycle was self-reported, the study design successfully allowed for tests and re-tests to be in the same phase for most female participants, making it unlikely that results were skewed or biased by menstrual cycle.

Participants self-selected into the SAUNA or CON groups. However, participants in both groups were given performance feedback following temperate exercise tests, which they discussed with their coach and used to inform their training. Given the highly competitive nature of the athletes recruited and the training ethos of the running club they were recruited from, the SAUNA and CON groups were equally invested in their training and performance during the exercise tests. This is supported by the similar SAUNA and CON training profiles (intensity, frequency, type and duration). Furthermore, the objective nature of the $V \mathrm{O}_{2 \max }$ and lactate profile tests reduces the impact of extrinsic, motivational factors on temperate performance results.

Although alternative methods of lactate profile testing may be preferred for determining lactate threshold (i.e., maximal lactate steady-state, or the D-max method described by Cheng et al. 1992), these methods significantly underestimate 'race pace' for $\sim$ 5-km races (Palmer et al. 1999; Chalmers et al. 2015). The training aims of the running club that participants were recruited from for the current study were largely centred around cross-country races (typically ranging between 4 and $6 \mathrm{~km}$ ). Thus, running speed at $4 \mathrm{mmol} \mathrm{L}^{-1}$ $\left[\mathrm{La}^{-}\right]$, observed to closely reflect 5-km race pace (Fay et al. 1989), had important ecological relevance for this cohort. In addition, though the results of this study certainly imply an improved exercise capacity, the extent at which the observed adaptations would impact self-paced performance in either the heat or in temperate conditions is unknown.
Finally, the repeated-measures aspect of the two participants who undertook both the SAUNA and CON intervention are not statistically accounted for. However, as depicted in figures where individual data from the crossover participant(s) are indicated, this exerted a negative bias for most measures. A sensitivity analysis was undertaken whereby the cross-over data for the two cross-over participants was removed (e.g., participants' data from the intervention they completed first were only included, and data from their cross-over intervention were removed; see supplementary material).

\section{Conclusion}

To the best of our knowledge, this study is the first to demonstrate that post-exercise sauna bathing can induce thermoregulatory adaptations during exercise at an absolute fixed-workload, which supports current recommendations to use post-exercise sauna bathing as an alternative to active heat acclimation (Racinais et al. 2019). These adaptations occur following 10 intermittent exposures across three weeks. Extending the intervention to seven weeks appeared to have little additional benefit. Further, this study supports the efficacy of heat acclimation as an ergogenic aid for exercise performance in temperate conditions. Specifically, intermittent post-exercise sauna bathing on average improved $V$ $\mathrm{O}_{2 \max }$ by $\sim 8 \%$, running speed at $4 \mathrm{mmol} \mathrm{L}^{-1}\left[\mathrm{La}^{-}\right]$by $\sim 4 \%$, and time to exhaustion by $\sim 12 \%$, all of which were significantly greater improvements than those exhibited by the CON group. This intermittent-style intervention is especially attractive as the flexible nature and minimal impact on normal training circumvent common challenges associated with more traditional active heat acclimation approaches.

Acknowledgements The authors thank Aidan Daniel, Patrick Harrison, Lucy Glover, Eleanor Anderson, Gabrielle Vickers and James Gibbon for their critical involvement in collection of experimental data, and Gabrielle Vickers for analysis of data for the purpose of generating feedback for participants. The authors thank all participants for their time and effort in the completion of this study.

Author contributions Authors NVK, SJEL, OJA and RAIL contributed to the conception and design of the study. All authors were involved in data acquisition, analysis, and interpretation of the data. NVK drafted the manuscript with all authors involved in the revision and approval of the final version of the manuscript.

Funding This study was funded by the University of Birmingham.

Data availability All data generated or analysed during this study on which the conclusions of the paper rely are included in this published article (and its supplementary information files). 


\section{Compliance with ethical standards}

Conflict of interest The authors declare that the research was conducted in the absence of any commercial or financial relationships that could be construed as a potential competing interest.

Open Access This article is licensed under a Creative Commons Attribution 4.0 International License, which permits use, sharing, adaptation, distribution and reproduction in any medium or format, as long as you give appropriate credit to the original author(s) and the source, provide a link to the Creative Commons licence, and indicate if changes were made. The images or other third party material in this article are included in the article's Creative Commons licence, unless indicated otherwise in a credit line to the material. If material is not included in the article's Creative Commons licence and your intended use is not permitted by statutory regulation or exceeds the permitted use, you will need to obtain permission directly from the copyright holder. To view a copy of this licence, visit http://creativecommons.org/licenses/by/4.0/.

\section{References}

Alber-Wallerström B, Holmer I (1985) Efficiency of sweat evaporation in unacclimatized man working in a hot humid environment. Eur J Appl Physiol 54(5):480-487

Borg GA (1982) Psychophysical bases of perceived exertion. Med Sci Sports Exerc 14(5):377-381

Casadio JR, Kilding AE, Cotter JD, Laursen PB (2017) From lab to real world: heat acclimation considerations for elite athletes. Sports Med 47(8):1467-1476. https://doi.org/10.1007/s4027 9-016-0668-9

Chalmers S, Esterman A, Eston R, Norton K (2015) Standardization of the Dmax method for calculating the second lactate threshold. Int J Sport Physiol 10(7):921-926

Chapman RF (2013) The individual response to training and competition at altitude. Brit J Sport Med 47(Suppl 1):i40-i44

Cheng B, Kuipers H, Snyder AC, Keizer HA, Jeukendrup A, Hesselink M (1992) A new approach for the determination of ventilatory and lactate thresholds. Sports Med 13(07):518-522

Cohen J (1988) Statistical power analysis for the behavioral sciences. Routledge, London

Edvardsen E, Hem E, Anderssen SA (2014) End criteria for reaching maximal oxygen uptake must be strict and adjusted to sex and age: a cross-sectional study. PLoS ONE. https://doi.org/10.1371/ journal.pone. 0085276

Fay L, Londeree BR, LaFontaine TP, Volek MR (1989) Physiological parameters related to distance running performance in female athletes. Med Sci Sport Exerc 21(3):319-324

Febbraio M, Snow R, Stathis C, Hargreaves M, Carey M (1994) Effect of heat stress on muscle energy metabolism during exercise. $\mathrm{J}$ Appl Physiol 77(6):2827-2831

Gagge AP, Stolwijk JAJ, Hardy JD (1967) Comfort and thermal sensations and associated physiological responses at various ambient temperatures. Environ Res 1(1):1-20. https://doi. org/10.1016/0013-9351(67)90002-3

Gagnon D, Ganio MS, Lucas RAI, Pearson J, Crandall CG, Kenny GP (2012) Modified iodine-paper technique for the standardized determination of sweat gland activation. J Appl Physiol 112(8):1419-1425. https://doi.org/10.1152/japplphysiol.01508 .2011

Garrett AT, Goosens NG, Rehrer NJ, Patterson MJ, Cotter JD (2009) Induction and decay of short-term heat acclimation. Eur $\mathbf{J}$ Appl Physiol 107(6):659-670. https://doi.org/10.1007/s0042 1-009-1182-7
Gerrett N, Kingma BRM, Sluijter R, Daanen HAM (2019) Ambient conditions prior to Tokyo 2020 Olympic and Paralympic games: considerations for acclimation or acclimatization strategies. Front Physiol 10:414. https://doi.org/10.3389/fphys.2019.00414

Giacomoni M, Bernard T, Gavarry O, Altare S, Falgairette G (2000) Influence of the menstrual cycle phase and menstrual symptoms on maximal anaerobic performance. Med Sci Sport Exerc 32(2):486-492. https://doi.org/10.1097/00005768-20000 2000-00034

Girard O, Amann M, Aughey R, Billaut F, Bishop DJ et al (2013) Position statement-altitude training for improving team-sport players' performance: current knowledge and unresolved issues. Br J Sports Med 47(Suppl 1):i8-16. https://doi.org/10.1136/bjspo rts-2013-093109

Griefahn B (1997) Acclimation to three different hot climates with equivalent wet bulb globe temperatures. Ergonomics 40(2):223234. https://doi.org/10.1080/001401397188314

Hafen PS, Preece CN, Sorensen JR, Hancock CR, Hyldahl RD (2018) Repeated exposure to heat stress induces mitochondrial adaptation in human skeletal muscle. J Appl Physiol 125(5):1447-1455

Howley ET, Bassett DR Jr, Welch HG (1995) Criteria for maximal oxygen uptake: review and commentary. Med Sci Sports Exerc 27(9):1292-1301

Ichinose TK, Inoue Y, Hirata M, Shamsuddin AKM, Kondo N (2009) Enhanced heat loss responses induced by short-term endurance training in exercising women. Exp Physiol 94(1):90-102

Jones AM, Doust JH (1996) A 1\% treadmill grade most accurately reflects the energetic cost of outdoor running. J Sport Sci 14(4):321-327. https://doi.org/10.1080/02640419608727717

Kirwan JP, Costill DL, Kuipers H, Burrell MJ, Fink WJ, Kovaleski JE, Fielding RA (1987) Substrate utilization in leg muscle of men after heat acclimation. J Appl Physiol 63(1):31-35

Lee BJ, Miller A, James RS, Thake CD (2016) Cross acclimation between heat and hypoxia: heat acclimation improves cellular tolerance and exercise performance in acute normobaric hypoxia. Front Physiol. https://doi.org/10.3389/fphys.2016.00078

Leppäluoto J, Tuominen M, Vaananen A, Karpakka J, Vuori J (1986) Some cardiovascular and metabolic effects of repeated sauna bathing. Acta Physiol Scand 128(1):77-81. https://doi. org/10.1111/j.1748-1716.1986.tb07952.x

Lorenzo S, Halliwill JR, Sawka MN, Minson CT (2010) Heat acclimation improves exercise performance. J Appl Physiol 109(4):1140 1147. https://doi.org/10.1152/japplphysiol.00495.2010

Matsumoto T, Kosaka M, Yamauchi M, Nakamura K, Yang GJ, Velazquez JJA (1990) Seasonal variation of thermal sweating. 熱 帯医学 Trop Med 32(2):73-80

McGarr GW, Hartley GL, Cheung SS (2014) Neither short-term sprint nor endurance training enhances thermal response to exercise in a hot environment. J Occup Environ Hyg 11(1):47-53

Mee JA, Doust J, Maxwell NS (2015) Repeatability of a running heat tolerance test. J Therm Biol 49-50:91-97. https://doi. org/10.1016/j.jtherbio.2015.02.010

Minson CT, Cotter JD (2016) CrossTalk proposal: heat acclimatization does improve performance in a cool condition. J Physiol 594(2):241

Nadel ER, Pandolf KB, Roberts MF, Stolwijk JA (1974) Mechanisms of thermal acclimation to exercise and heat. J Appl Physiol 37(4):515-520

Novak D, Tudor PB, Matkovic BR (2018) Adding sauna bathing after endurance training: a practical insight from the word's top junior tennis player. Acta Kinesiologica 12(1):50-54

Palmer A, Potteiger J, Nau K, Tong R (1999) A 1-day maximal lactate steady-state assessment protocol for trained runners. Med Sci Sports Exerc 31(9):1336-1341

Périard JD, Racinais S, Sawka MN (2015) Adaptations and mechanisms of human heat acclimation: applications for competitive 
athletes and sports. Scand J Med Sci Sports 25(Suppl 1):20-38. https://doi.org/10.1111/sms.12408

Płoszczyca K, Langfort J, Czuba M (2018) The effects of altitude training on erythropoietic response and hematological variables in adult athletes: a narrative review. Front Physiol 9:375. https:// doi.org/10.3389/fphys.2018.00375

Racinais S, Alonso JM, Coutts AJ, Flouris AD, Girard O et al (2015) Consensus recommendations on training and competing in the heat. Sports Med 45(7):925-938. https://doi.org/10.1007/s4027 9-015-0343-6

Racinais S, Casa D, Brocherie F, Ihsan M (2019) Translating Science Into Practice: The Perspective of the Doha 2019 IAAF World Championships in the Heat. Frontiers in Sports and Active Living 1

Racinais S, Periard JD, Karlsen A, Nybo L (2015) Effect of heat and heat acclimatization on cycling time trial performance and pacing. Med Sci Sport Exer 47(3):601-606. https://doi.org/10.1249/ Mss.0000000000000428

Ramanathan NL (1964) New weighting system for mean surface temperature of human body. J Appl Physiol 19(3):531-540

Rønnestad BR, Hamarsland H, Hansen J, Holen E, Montero D, Whist JE, Lundby C (2020) Five weeks of heat training increases hemoglobin mass in elite cyclists. Exp Physiol 1-12. https://doi. org/10.1113/EP088544

Rusko HK, Tikkanen HO, Peltonen JE (2004) Altitude and endurance training. J Sport Sci 22(10):928-944. https://doi. org/10.1080/02640410400005933

Sawka MN, Burke LM, Eichner ER, Maughan RJ, Montain SJ, Stachenfeld NS (2007) Exercise and fluid replacement. Med Sci Sport Exerc 39(2):377-390. https://doi.org/10.1249/mss.0b013 e31802ca597

Sawka MN, Leon LR, Montain SJ, Sonna LA (2011) Integrated physiological mechanisms of exercise performance, adaptation, and maladaptation to heat stress. Compr Physiol 1(4):1883-1928. https://doi.org/10.1002/cphy.c100082
Scoon GS, Hopkins WG, Mayhew S, Cotter JD (2007) Effect of postexercise sauna bathing on the endurance performance of competitive male runners. J Sci Med Sport 10(4):259-262. https://doi. org/10.1016/j.jsams.2006.06.009

Smith CJ, Havenith G (2019) Upper body sweat mapping provides evidence of relative sweat redistribution towards the periphery following hot-dry heat acclimation. Temperature 6(1):50-65

Stanley J, Halliday A, D’Auria S, Buchheit M, Leicht AS (2015) Effect of sauna-based heat acclimation on plasma volume and heart rate variability. Eur J Appl Physiol 115(4):785-794. https://doi. org/10.1007/s00421-014-3060-1

Tamura Y, Matsunaga Y, Masuda H, Takahashi Y, Takahashi Y et al (2014) Postexercise whole body heat stress additively enhances endurance training-induced mitochondrial adaptations in mouse skeletal muscle. Am J Physiol Reg I 307(7):R931-R943. https:// doi.org/10.1152/ajpregu.00525.2013

Tyler CJ, Reeve T, Hodges GJ, Cheung SS (2016) The effects of heat adaptation on physiology, perception and exercise performance in the heat: a meta-analysis. Sports Med 46(11):1699-1724. https:// doi.org/10.1007/s40279-016-0538-5

van Gent RN, Siem D, van Middelkoop M, Van Os AG, Bierma-Zeinstra SMA, Koes BW (2007) Incidence and determinants of lower extremity running injuries in long distance runners: a systematic review. British J Sports Med 41(8):469-480

Vickers AJ, Altman DG (2001) Analysing controlled trials with baseline and follow up measurements. BMJ 323(7321):1123-1124

Young AJ, Sawka MN, Levine L, Cadarette BS, Pandolf KB (1985) Skeletal-muscle metabolism during exercise is influenced by heat acclimation. J Appl Physiol 59(6):1929-1935

Publisher's Note Springer Nature remains neutral with regard to jurisdictional claims in published maps and institutional affiliations. 